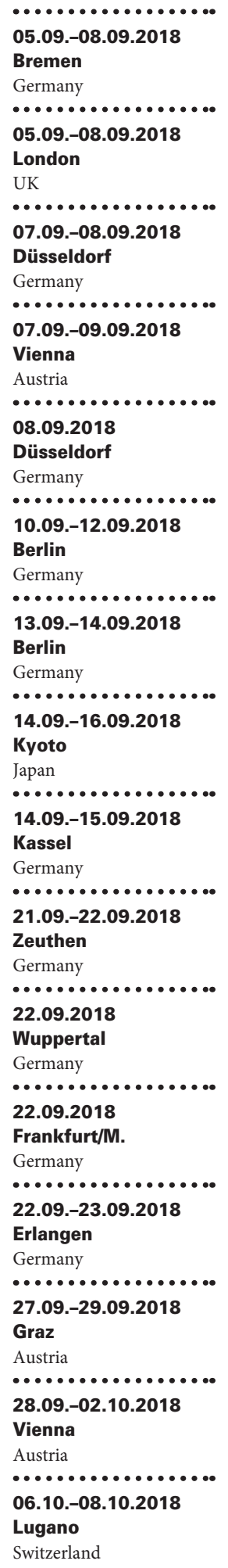

12. Kongress der Deutschen Gesellschaft für Palliativmedizin

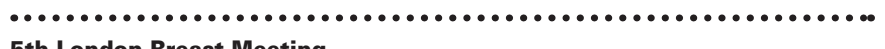

5th London Breast Meeting

10. Wissenschaftliches Symposium der Kommission Translationale Forschung der Arbeitsgemeinschaft Gynäkologische Onkologie

ECCO 2018: European Cancer Summit

7. Düsseldorfer Gynäkologisches Sommersymposium

21. Repetitorium für Gynäkologie und Geburtshilfe

Master Class Breast Surgery

17th Biennial Meeting of the International Gynecologic Cancer Society

\section{AGO-Zertifizierungskurs Gynäko-Onkologie}

Tumorpatienten bestmöglich versorgen - Auffrischkurs Onkologie

Wild West Symposium

12. Rhein-Main-Symposium

21. Mammasonographiekurs Erlangen

Jahrestagung der Österreichischen Gesellschaft für Senologie

Jahrestagung der Deutschen, Österreichischen und Schweizerischen Gesellschaften für Hämatologie und Medizinische Onkologie

4th ESO-ESMO Breast Cancer in Young Women International Conference (BCY4)
Information: www.dgp2018.de

Information: oncoplasticbc.org/

Information: www.if-kongress.de

Information: www.ecco-org.eu

Information: www.medical-communications.de/home

Information: www.charite-repetitorium.de

Information: www.eickeler.org

Information: www.igcs2018.com

Information: www.medconcept.org

Information: www.medconcept.org

Information: www.wild-west-symposium.de

Information: mci-academy.de/rms2018/

Information: www.radiologie.uk-erlangen.de/aktuelles/ veranstaltungen

Information: www.medacad.org/senologie2018/

Information: www.haematologie-onkologie-2018.com

Information: http://bit.ly/2D47SIg

\section{KARGER}

Fax +497614520714

Information@Karger.com

www.karger.com

\section{() 2018 S. Karger GmbH, Freiburg}




\section{•................}

11.10.-13.10.2018

Istanbul

Turkey

•.................

12.10.-13.10.2018

Kampen/Sylt

Germany

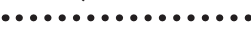

12.10.-13.10.2018

Wiesbaden

Germany

•...............

19.10.-23.10.2018

Munich

Germany

00000000000000.00

31.10.-03.11.2018

Berlin

Germany

•.............

09.11.-10.11.2018

Kassel

Germany

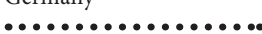

10.11 .2018

UIm

Germany

.................

14.11.-16.11.2018

Basel

Switzerland

.................

21.11.2018

Essen

Germany

...............

22.11.-23.11.2018

Cologne

Germany

.................

23.11.-24.11.2018

Lübeck

Germany

24.11.2018

Cologne

Germany

•................

29.11.-30.11.2018

Tübingen

Germany

..................

30.11.-01.12.2018

Kassel

Germany

01.12 .2018

Krefeld

Germany

...................

04.12.-08.12.2018

San Antonio, TX

USA

.................

15.12.2018

Munich

Germany

16.01.20...

Frankfurt/M.

Germany

19.01 .2019

Stuttgart

Germany 3rd Breast Cancer Conference. Breastanbul

5. Sylt-Refresher Gynäkologischer Ultraschall

Zertifikat Integrative Onkologie - Modul Oktober 2018: Ernährung

ESMO 2018 Congress - Securing Access to Optimal Cancer Care

62. Kongress der Deutschen Gesellschaft für Gynäkologie und

\section{Geburtshilfe}

AGO-Zertifizierungskurs Gynäko-Onkologie

5. Ulmer Herbstsymposium - Senologie und Gynäkologische Onkologie Update

42. Dreiländertreffen der DEGUM, ÖGUM, SGUM

Best of Europe: State of the Art bei soliden Tumoren. Aktuelles vom

Europäischen Krebskongress

Master Class Breast Surgery

21. Ostsee-Seminar

8. Rhein-Ruhr-Symposium

8. Tübinger Brustchirurgische Tage

AGO-Zertifizierungskurs Gynäko-Onkologie

VI. Niederrhein-Kolloquium 2018 - Update Senologie und

Gynäkoonkologie für Klinik und Praxis

39th Annual San Antonio Breast Cancer Symposium 2018

12. Wintersymposium München: Gynäkologische Tumoren und Brustkrebs - aktuelle Entwicklungen

Frankfurter Winter-Fortbildung: Post San Antonio

17. Tübinger Airport Meeting - Update Gynäkologische Onkologie
Information: www.breastanbul.org

Information: www.sylt-refresher.de

Information:

http://prio-dkg.de/fortbildungen-und-veranstaltungen/

Information:

www.esmo.org/Conferences/ESMO-2018-Congress

Information: www.dggg2018.de

Information: www.medconcept.org

Information: www.medical-communications.de/home

Information: ultraschall2018.ch

Information: www.eickeler.org

Information: www.eickeler.org

Information: www.if-kongress.de/veranstaltungen

Information: www.rhein-ruhr-symposium.info

Information: www.if-kongress.de/veranstaltungen

Information: www.medconcept.org

Information: www.medical-communications.de/home

Information: www.sabcs.org/2018-SABCS

Information: www.wintersymposium-muenchen.de

Information:

www.rhein-main-gyn.de/save-the-date-nac.html

Information: www.if-kongress.de/veranstaltungen 
08.0.................

Munich

Germany

09.02 .2019

Munich

Germany

16.02 .2019

Essen

Germany

...............

22.02.-23.02.2019

Potsdam

Germany

19.03.2019
Vienna
Austria
$\ldots \ldots \ldots \ldots \ldots \ldots . . \ldots \ldots \ldots . . . \ldots$

20.03.-23.03.2019

Vienna

Austria

[..................

22.03.-23.03.2019

Stuttgart

Germany

................

28.03.-29.03.2019

Berlin

Germany

.................

28.03.-29.03.2019

Hamburg

Germany

...............

03.04.-06.04.2019

Berlin

Germany

..................

02.05.-04.05.2019

Berlin

Germany

•................

09.05.-11.05.2019

Munich

Germany

.................

12.06.2019

Frankfurt/M.

Germany

.................

27.06.-29.06.2019

Kampen/Sylt

Germany

•..............

27.06.-29.06.2019

Berlin

Germany

.................

05.09.-07.09.2019

Essen

Germany

•...............

16.10.-19.10.2019

Mainz

Germany

[................

25.10.-26.10.2019

Würzburg

Germany

•....................

17.06.-20.06.2020

Torino

Italy
AWOgyn Jahrestagung

Information: www.if-kongress.de/veranstaltungen

11. Jahreskongress des Tumorzentrums München (TZM Essentials 2019)

9. Essener Symposium zur gynäkologischen Onkologie und Senologie

6. Brandenburger Krebskongress

4th Biannual Vienna Breast Surgery Day

16th St. Gallen International Breast Cancer Conference

Gynäkologie und Geburtshilfe aktuell

Interdisziplinärer Kongress Quality of Cancer Care 2019 (QoCC 2019)

16. Hamburger Update Gynäkologie und Geburtshilfe

10. International Charité-Mayo-Conference - Updates in Gynecology: A Global Perspective

\section{ESMO Breast Cancer Annual Congress}

AGO-Symposium - State of the Art: Kongress Gynäkologische Onkologie

Neues aus Chicago

8. Sylter Fortbildungstage Frauenheilkunde und Geburtshilfe

39. Jahrestagung der Deutschen Gesellschaft für Senologie

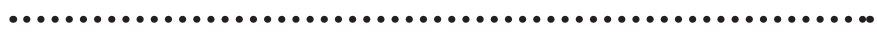

Master of Disaster: Komplikationsmanagement in der Senologie und Gynäkologischen Onkologie

43. Dreiländertreffen der DEGUM, ÖGUM, SGUM

3. Franken Fortbildung Frauengesundheit

26th Biennial Congress of the European Association for Cancer Research
Information: www.tzm-essentials.de

Information: www.eickeler.org

Information: www.mcall-gmbh.de/bb-krebskongress/

Information: http://www.vbsd.at

Information: www.oncoconferences.ch

Information: www.gyn-geb-aktuell.de

Information: www.qocc.de

Information: www.hamburger-update.de

Information: www.charite-mayo.de

Information:

www.esmo.org/Conferences/ESMO-Breast-Cancer-2019

•................................

Information: www.if-kongress.de/veranstaltungen

Information: www.eickeler.org

Information: www.gynsylt.de

Information: www.senologiekongress.de

Information: www.masterofdisaster.org

Information:

http://www.degum.de/aktuelles/veranstaltungen.html

Information: www.if-kongress.de/veranstaltungen

Information: https://www.eacr25.org/ 